\title{
Origine géographique et sociale des étudiants du petit séminaire de Chicoutimi et leur orientation socio-professionnelle : 1873-1930
}

Jean-Paul Simard et Bérard Riverin

Volume 40, 1973

URI : https://id.erudit.org/iderudit/1007247ar

DOI : https://doi.org/10.7202/1007247ar

Aller au sommaire du numéro

Éditeur(s)

Les Éditions Historia Ecclesiæ Catholicæ Canadensis Inc.

ISSN

0318-6172 (imprimé)

1927-7067 (numérique)

Découvrir la revue

Citer cet article

Simard, J.-P. \& Riverin, B. (1973). Origine géographique et sociale des étudiants du petit séminaire de Chicoutimi et leur orientation socio-professionnelle :

1873-1930. Sessions d'étude - Société canadienne d'histoire de l'Église catholique, 40, 33-53. https://doi.org/10.7202/1007247ar

Tous droits réservés @ Les Éditions Historia Ecclesiæ Catholicæ Canadensis Inc., 1974
Ce document est protégé par la loi sur le droit d'auteur. L'utilisation des services d’Érudit (y compris la reproduction) est assujettie à sa politique d'utilisation que vous pouvez consulter en ligne. 


\section{Origine géographique et sociale des étudiants du petit séminaire de Chicoutimi et leur orientation socio-professionnelle: 1873-1930}

I

Après avoir pris connaissance de notre travail, certains se demanderont peut-être, d'abord si ce sujet n'aurait pas mieux convenu à des sociologues, des démographes ou des statisticiens, plutôt qu'à des historiens. D'autres pourront également s'interroger sur les raisons qui ont motivé notre choix.

La pertinence de ces questions ne fait évidemment aucun doute. La définition du travail de l'historien apporte un élément de réponse à la première interrogation. En effet, de plus en plus, se lèvent les barrières entre les différentes disciplines, et on laisse à l'historien le rôle qui lui revient, c'est-à-dire la redécouverte de l'homme à l'intérieur des sociétés. On s'accorde donc aujourd'hui sur le fait qu'il revient à ce chercheur, non seulement de dépouiller des documents afin d'en tirer des renseignements, mais aussi de procéder à leur analyse et à leur interprétation en situant les phénomènes historiques dans leurs cadres sociaux. Or, on peut dire que notre travail et nos préoccupations se situent précisément dans cette ligne de pensée.

En réponse à la seconde question, disons que ce n'est toutefois qu'après un sérieux examen de nos possibilités et de nos préoccupations que s'est manifesté notre intérêt pour un sujet de cette nature. Et le facteur qui fut prépondérant dans notre choix est sans aucun doute l'importance de l'éducation et de la culture dans notre société moderne, rôle qu'on qualifie parfois de principal facteur d'intégration et de promotion sociale.

L'éducation peut ignorer, en effet, deux rôles principaux dans la société. D'une part, elle sert à perpétuer et à étendre la culture; d'autre part, elle peut, en s'ouvrant à une partie plus ou moins étendue de la population, contribuer à affaiblir ou à affermir les véritables fondements de la stratification sociale.

Du fait que la culture et l'instruction ont été, au Québec, diffusées fort longtemps par des institutions religieuses, nous avons été amenés à nous interroger sur le rôle joué par le Séminaire de Chicoutimi dans la 
société saguenéenne. Nous avons donc voulu voir si ce collège classique s'était donné comme but de transmettre une culture et, par là, de conserver un système de valeurs, ou simplement de perpétuer une hiérarchie sociale en assurant le remplacement d'une certaine élite. Par là, nous espérions mesurer l'impact social et culturel qu'a pu avoir une institution de ce genre dans une société naissante. C'est tout autour de cette problématique que s'est défini le but de notre recherche.

Nous sommes donc partis de l'hypothèse que, pendant la période de 1873-1905, période qui voit la fondation du Séminaire et son intégration dans les' cadres de la société saguenéenne, l'institution se serait surtout employée à poursuivre des tâches culturelles, assurant ainsi la continuité du système de valeurs déjà en place dans la province et la relève des prêtres dont avait besoin l'Église diocésaine et que nécessitait le désir d'encadrement religieux de notre jeune société régionale. Le Séminaire procédera alors à une certaine diversification de son recrutement.

La période qui suit, celle de 1906-1930, verrait le Séminaire, maintenant assuré de son existence, sans changer d'objectifs, procéder à une certaine réévaluation des fins qu'il poursuit. À sa tâche culturelle initiale, il aurait greffé certaines préoccupations d'ordre social, face aux besoins nouveaux émergeant de la communauté régionale. Le Saguenay de 1906 connait une première expansion industrielle, c'est l'heure de l'industrie des pâtes et papiers: deux moulins produisent à Chicoutimi même; Jonquière, Val Jalbert et Péribonka possèdent le leur. Une classe proprement ouvrière apparaît alors dans le paysage saguenéen, avec ses problèmes, ses difficultés et ses aspirations. Si tel fut le cas, l'institution se serait davantage souciée, à partir du début du $\mathrm{XX}^{e}$ siècle, de développer les élites laïques destinées à assurer le leadership socio-économique de la société saguenéenne.

Enfin, pendant ces 57 premières années de son existence, le Séminaire de Chicoutimi a très bien pu manifester une quelconque préférence envers certains éléments de la société qui démontraient plus de dispositions aux objectifs et aux idéaux auxquels il aspirait. Voilà, résumée en quelques mots, toute la problématique que nous désirions mettre à l'épreuve. Notons toutefois que nous ne prétendons aucunement vider un sujet aussi complexe, ce que l'état actuel de nos recherches ne nous permet d'ailleurs pas. Nous nous contentons, par cet exposé, d'étaler, sans plus, quelques résultats, qui sont encore loin d'être définitifs, d'une enquête plus vaste et plus poussée.

Dès le début, nous étions pleinement conscients des difficultés auxquelles nous aurions à faire face durant le cheminement de notre enquête. Aussi, nous sommes-nous efforcés de ne pas déroger aux règles de 
rigueur et d'objectivité qui doivent gouverner ce genre d'analyse. Nous tenions également à ce que nos conclusions intéressent, bien sûr, les historiens, mais tout autant les sociologues, les démographes et les géographes, de même tous ceux qui s'intéressent aux problèmes actuels de l'éducation. Voilà pourquoi nous avons tenté d'orienter notre recherche de façon à produire des données quantifiables et à exploiter le plus scientifiquement possible les documents que nous avions entre les mains.

\section{II}

\section{Les sources}

Avant d'aller plus loin, nous nous devons de décrire brièvement les principales sources que nous avons dépouillées:

1. Registres de l'iñscription des élèves du Séminaire de Chicoutimi, 1891. 1930.

Ces manuscrits sont les documents de base de notre recherche. Ils nous furent d'une extrême utilité pour dresser une échelle de l'origine sociale des étudiants et l'éventail complet de leur origine géographique. Ils contiennent le nom des élèves pour chaque année académique, leur âge, le nom de leur père et lieu de résidence.

2. Registre général des examens du Petit-Séminaire de Chicoutimi, 18781932.

Cette source nous a beaucoup servi à identifier les cas de succès et d'abandon de chaque étudiant qui fréquenta l'institution pendant la période.

3. Les Annuaires reconstitués du Séminaire de Chicoutimi, 1873-1880.

Ces annuaires ont été colligés par les soins de M. Léonidas Larouche, archiviste au Séminaire. Ils contiennent la liste annuelle des élèves, le degré académique, le nom du père et parfois son lieu de résidence.

4. La liste des anciens élèves du Séminaire de Chicoutimi, 1873-1940.

Cette liste dressée par M. Georges Renaud Pilote donne le nom de l'étudiant, son orientation professionnelle, l'année terminale de son cours soit comme finissant ou comme cas d'abandon.

5. Les Annuaires du Séminaire de Chicoutimi, 1880-1930.

Cette source imprimée donne, pour chaque année, la liste des élèves qui fréquentent chacune des classes du Séminaire.

L'utilisation de ces principaux documents a permis une analyse assez approfondie des sujets suivants: le recrutement géographique et la 
provenance des étudiants du Séminaire, les cas de réussite et d'abandon en regard de l'appartenance socio-professionnelle et, enfin, l'orientation de chacun des finissants.

Nous tenons ici à remercier particulièrement le Séminaire de Chicoutimi de sa bienveillance et de l'intérêt qu'il a porté à notre travail en nous facilitant l'accès à ses archives. Nous nous en voudrions également de ne point souligner la collaboration de M. Gérard Bouchard, professeur à l'Université du Québec à Chicoutimi. Ses conseils pertinents nous ont souvent aidés dans la marche générale de cette recherche.

III

Nous allons donc, sans plus tarder, vous faire part des résultats obtenus relativement aux quatre points sur lesquels a porté notre enquête, à savoir: le recrutement géographique, la provenance sociale, la persévérance scolaire et, enfin, les choix de carrières des étudiants du Séminaire.

A. Les facteurs géographiques du recrutement (Cf. Carte géographique)

Concernant le recrutement géographique des élèves de l'institution, nous avons établi notre étude en fonction de sept grandes catégories correspondant chacune à une aire géographique régionale déterminée, soit Chicoutimi, Jonquière (Kénogami), la région de la Baie (Bagotville, GrandeBaie et Port-Alfred), le Saguenay non urbain, la région du Lac Saint-Jean, Charlevoix et les "Autres régions». Il nous a, en effet, paru inutile de détailler davantage l'origine géographique des élèves, étant donné que, d'une part, le nombre d'étudiants n'était pas tellement élevé, et que, d'un autre côté, souvent les registres d'inscription nous fournissaient l'origine régionale sans plus de précision (noms de villes, villages, paroisses, etc.). Également, nous sommes arrivés à constater qu'une subdivision de la sorte répondait de façon satisfaisante à nos aspirations et remplissait assez bien le rôle que nous lui avions assigné au départ.

Tenant compte de ces divers facteurs, nous avons donc considéré:

1) La concentration démographique de Chicoutimi: la ville (village jusqu'en 1882), et ses alentours assez immédiats, prise à part, vu sa situation privilégiée par rapport au Séminaire et l'importance qu'elle a eue dans l'alimentation en effectifs étudiants de l'institution.

2) Nous avons également tenu à considérer distinctement d'autres centres qui, même si à l'époque de la fondation du Séminaire, étaient encore très peu importants, sont devenus plus tard des centres de recrutement non négligeables. Ainsi, nous avons isolé, en tant que bassins spécifiques du recrutement géographique, tout d'abord Jonquière 
(Kénogami) et ensuite la région de la Baie (Bagotville, Grande-Baie, et Port-Alfred).

3) La région du Saguenay non urbain (i.e. le territoire compris de chaque côté des deux rives entre Saint-Ambroise et Petit-Saguenay inclusivement, exceptions faites de Chicoutimi, Jonquière (Kénogami) et de la Baie des $\mathrm{Ha}$ ! Ha!) a, elle aussi, été considérée spécifiquement dans notre analyse du recrutement.

4) La région du Lac Saint-Jean ( les contours du Lac à partir de SaintBruno inclusivement).

5) La région de Charlevoix, comprenant le territoire allant de SaintFirmin et l'Île-aux-Coudres jusqu'à Baie Sainte-Catherine.

6) Enfin, une catégorie que nous avons convenu d'appeler "Autres régions", composée de régions situées totalement en dehors de l'aire géographique de recrutement du Séminaire (ex.: le reste de la province de Québec, le Canada, les États-Unis, etc.).

Une subdivision géographique de ce genre nous fait aboutir à des proportions quand même assez révélatrices quant à l'origine régionale des étudiants (cf. Tableau I).

L'étude de ce tableau nous amène aux constatations suivantes : nous remarquons tout d'abord une certaine diversification géographique du recrutement des élèves, diversification qui tend à s'accentuer avec la seconde période (1906-1930). Le Séminaire semble donc par là avoir fait un effort pour régionaliser son emprise et, par le fait même, sa vocation culturelle.

En deuxième lieu, le graphique nous permet de constater que, pendant la seconde période étudiée (1906-1930), la percée de l'institution en milieu rural se poursuit en s'accentuant. Par exemple: le cas de la région du Lac Saint-Jean pour qui, durant la première période (1873-1905), la participation à la composition de la population étudiante du Séminaire était de $12.2 \%$, passe, de 1906-1930, à $34.1 \%^{1}$. Également, le cas des régions du Saguenay non urbain et de Charlevoix pour lesquelles en 1930 la population est rurale respectivement à $96 \%$ et $72 \%$ est à remarquer ${ }^{2}$. Ces deux régions, même si elles connaissent de légères baisses proportionnelles par rapport au recrutement, réalisent toutefois de fortes hausses en chiffres absolus. environ.

A noter qu'en 1931 la population du lac Saint-Jean est encore rurale à $72 \%$

2 Ces compilations ont été faites d'après les chiffres des recensements fédéraux correspondant à la période étudiée. 
Par l'étude de ce tableau, nous nous sommes également demandé si le Séminaire ne se serait pas détourné de la clientèle urbaine pour la période étudiée (1873-1930). Les graphiques nous montrent que les territoires urbains participent pour peu à la composition de la clientèle de l'institution (ex.: Jonquière-Kénogami; le secteur de la Baie des Ha! Ha!, etc.).

Mais ce qui toutefois demeure le plus frappant, c'est la faible hausse (en nombre absolu) que connaît Chicoutimi pour la seconde période et, surtout, la baisse qu'elle accuse en nombres relatifs ${ }^{3}$. Un test que nous avons effectué nous a révélé, après comparaison entre les deux périodes, un indice d'augmentation de la population de Chicoutimi de 3.7 par rapport à un indice d'augmentation de seulement 1.5 quant à sa participation à la composition de la clientèle de l'institution.

Une dernière comparaison a été établie entre l'indice de croissance démographique des régions de Chicoutimi, Saguenay, Lac Saint-Jean et Charlevoix et l'indice de l'augmentation de leur participation à la composition de la population étudiante du Séminaire. Le résultat du test démontre un indice d'accroissement démographique total (pour ces quatre régions) de 2.2, par rapport à un indice d'augmentation du recrutement de 2.6. Ce qui nous montre assez clairement que le mouvement de scolarisation engendré par le Séminaire a même débordé le mouvement de la population et nous porte à croire que l'institution a fait de réels efforts pour accomplir sa mission éducatrice.

\section{B. La provenance sociale des étudiants (Cf. Tableau II)}

En ce qui a trait au recrutement de la clientèle de l'institution, disons que, en collaboration avec M. Gérard Bouchard, professeur à l'université du Québec à Chicoutimi, nous avons mis au point une échelle socioprofessionnelle qui, tout en étant utile, appelle toutefois quelques remarques et nous amène de ce fait à formuler des mises en garde, voire certaines réserves (cf. Grille socio-professionnelle).

Il est très important au départ, afin d'éviter le plus grand nombre possible de risques de confusion, de porter notre attention sur le fait que cette échelle repose sur une classification socio-professionnelle des occupations et non pas socio-économique. De ce fait, nous nous sommes abstenus autant que possible, lors de son établissement, de recourir à des définitions relativement à des secteurs particuliers de l'activité économique tels agriculture, forêt, services, etc. ${ }^{4}$.

3 La clientèle que Chicoutimi fournit passe de 135 à 200 élèves, mais, également, de $36.7 \%$ de la composition totale, elle baisse à $22.4 \%$.

4 Cérard Bouchard, Démographie et société au Saguenay, le cas de Laterrière: 18551969. Communication au Congrès de la Société Historique du Canada, Kingston, 1973 (texte d'introduction, p. 6-7). 
Nous nous sommes donc surtout attachés à définir socialement et de façon empirique chaque catégorie en la référant à la somme d'autorité, de prestige et de pouvoir qui y était rattachée ${ }^{5}$. Même si, de prime abord, cette façon de procéder semble donner libre cours à certaines ambiguïtés (ce dont nous sommes pleinement conscients), elle nous paraissait au départ suffisante pour notre étude et nous évitait par là un long recours à d'autres sources documentaires qui auraient servi à la rendre plus exhaustive, plus précise.

N'omettons pas non plus d'ajouter que la mise au point de cette échelle socio-professionnelle nous a contraints à procéder à des choix par trop souvent intuitifs que d'aucuns pourront même qualifier de discutables. Pour cette raison, et afin d'éviter toute la confusion et l'arbitraire qu'une trop grande tentative de précision n'aurait pas manqué d'apporter, nous avons décidé en dernier recours l'utilisation de termes aussi généraux et imprécis que cultivateur, industriel, fabricant, etc. De ce fait également, nous nous sommes permis de procéder à des regroupements qui font se côtoyer sous une même appellation les cultivateurs de tous revenus; plus de précision nous aurait obligés à procéder à une compilation très précise des revenus de cette couche socioprofessionnelle.

Également, si, dans notre étude, les analyses et les conclusions portent surtout sur le groupe des fils de cultivateurs, cela s'explique par leur importance numérique dans la composition de la société saguenéenne et, conséquemment, dans la population étudiante du Séminaire.

Les autres catégories sociales prises individuellement ne représentaient souvent qu'un nombre insuffisant de cas, ne permettant pas, par là, d'avancer des conclusions absolument certaines. Afin d'obvier à cette difficulté et de donner à notre étude toute la signification possible, nous avons opéré des regroupements sectoriels mettant sous une même appellation plusieurs professions possédant des notes et caractéristiques communes.

Ainsi, nous retrouvons ensemble ouvriers spécialisés, ouvriers non spécialisés et travailleurs forestiers; ensuite, sous l'appellation "Classes moyennes": "artisans, petits entrepreneurs et fabricants" sont associés aux "métiers du commerce", aux "métiers tertiaires" et aux "petits notables"; enfin, dans une catégorie appelée "Élite économique et sociale ", se regroupent les "professions libérales" et les "industriels, financiers et gros entrepreneurs".

5 Ibid., p. 7. 
Disons enfin que nous n'avons jamais entretenu la prétention d'établir par cette classification quoi que ce soit de définitif. Mais cette échelle socio-professionnelle trouve sa raison d'être et sa réelle valeur en ce qu'elle répond assez justement aux fins que nous lui assignons ici.

La principale constatation qui peut se dégager du Tableau II est sans aucun doute la forte accession de la classe agricole à l'instruction classique. En effet, les fils de cultivateurs font figure de véritables privilégiés par rapport aux descendants des autres classes sociales, fournissant pour chacune des périodes (1873-1905 et 1906-1930) respectivement $39.2 \%$ et $32.9 \%$ des effectifs étudiants de l'institution.

Toutefois, le fait que cette catégorie sociale constitue à l'époque l'une des forces numériques les plus importantes de la société québécoise et, a fortiori, de la société saguenéenne, ne fournit pas, selon nous, une explication satisfaisante de ce phénomène. Cette particularité nous a poussés à formuler l'hypothèse suivante: le Séminaire, ayant cru trouver dans cette classe sociale une source considérable de vocations religieuses, a peut-être de ce fait favorisé son accès à l'institution. Alors, la classe rurale, répondant le mieux aux aspirations que manifestait à l'époque le collège classique de Chicoutimi, fut fort probablement encouragée à y inscrire ses enfants.

Disons enfin que, pour donner à ce tableau toute sa valeur significative, il aurait fallu établir une comparaison entre la composition sociale de la clientèle étudiante de l'institution et la composition socioprofessionnelle de chaque région considérée, ce qui s'est avéré absolument impossible. Cependant, nous avons pu, par la consultation des rôles d'évaluation, faire le test avec Chicoutimi, relativement à la période 1873 à 1905.

A partir des résultats obtenus, nous avons été en mesure de constater que, sur un total de 427 ouvriers (61\% de la composition sociale de la ville), 13 étudiants seulement représentaient cette classe au Séminaire. Tandis que les cultivateurs, sur un total de 23 (3.4\% de la composition sociale de Chicoutimi), étaient représentés par 11 de leurs fils au Séminaire et participaient ainsi pour $15.1 \%$ à la composition sociale de la population étudiante de l'institution. Ce qui est quand même significatif.

C. Les facteurs sociaux de la persévérance scolaire

1) L'origine sociale des finissants (cf. Tableau III)

Afin de bien saisir toute la valeur significative des données du Tableau III, il importe de toujours comparer le pourcentage de finissants de chaque catégorie sociale au pourcentage total des finissants. Cette précision faite, 
nous sommes en mesure de constater que, pour la première période ( 1873 . 1905), les fils de cultivateurs, représentant 39\% des étudiants inscrits, sont nettement sur-représentés chez les finissants, terminant leur cours dans une proportion de près de $70 \%$ (68.9).

Notons que, dans ce domaine également, les étudiants issus des classes de "l'élite" jouissent d'une importante sur-représentation. Évidemment, leur nombre restreint nous oblige à éviter d'étayer des conclusions.

Les "Ouvriers et les classes moyennes", pour leur part, ne semblent pas bénéficier d'autant de chance que les catégories précédentes, étant sous-représentés chez les finissants.

Donc, à l'instar de ce qu'avait laissé supposer l'hypothèse déjà énoncée, nous constatons que, étant les plus nombreux survivants aux rigueurs de la sélection et aux différentes autres exigences de l'institution, les étudiants issus de la classe qui entrent au Séminaire sont ceux qui semblent avoir le plus de chances de mener à bien leurs études classiques.

Pour la seconde période, les traits constatés antérieurement s'atténuent nettement. La classe agricole qui demeure sur-représentée chez les finissants voit quand même diminuer de façon sensible l'écart de sur-représentation. Par ailleurs, dans ce domaine, la situation des ouvriers, des classes moyennes et des élites se normalise.

Tous ces éléments observés pour la deuxième période (1906-1930) nous portent à croire que le Séminaire entre, à l'époque, dans une période de mutation et prépare peut-être une réorientation de ses objectifs.

2) Les cas d'abandon (cf. Tableaux IVa et IVb)

Pour la période étudiée, les cas d'abandon des études ont atteint une proportion nettement digne d'intérêt. En effet, le fait que $42 \%$ (pour la période de 1873-1905) et 54\% (pour la période de 1906-1930) des étudiants, pour une raison ou pour une autre, aient quitté l'institution dans le courant de leurs études, ne peut laisser indifférent. Il conviendrait ici de mentionner les principales causes d'abandon que nous sommes parvenus à retracer au cours de notre recherche.

Nous avons regroupé les motifs de départ en deux catégories principales, à savoir : les départs plus ou moins volontaires, en second lieu, les renvois proprement dits. On pourrait ranger dans le premier groupe les cas de maladie, les départs d'étudiants quittant la région pour aller poursuivre leurs études dans un autre collège, les dossiers scolaires peu reluisants, l'émigration et les difficultés monétaires. Cette dernière catégorie se présente sous deux formes: soit l'incapacité pour le père d'un élève de 
satisfaire aux exigences financières de l'institution ${ }^{6}$, soit le besoin de maind'œuvre pour le travail de la terre ou du petit commerce. Ainsi, plusieurs élèves issus de la classe agricole furent obligés de laisser leurs études afin d'aider leur père à pourvoir aux besoins de la famille. C'est en ce sens qu'on peut réellement associer l'abandon prématuré des études à la classe sociale.

En ce qui concerne les cas de renvoi, soulignons la question de mœurs, l'insubordination flagrante, la violation répétée des règlements, les échecs scolaires, etc.

Le tableau IV nous pousse à constater que le taux d'abandon des fils de cultivateurs pendant la première période (1873-1905) est beaucoup moins élevé que celui des autres groupes sociaux. Il nous est donc permis, une fois de plus, de penser que le Séminaire pouvait faire preuve d'une certaine indulgence à l'égard des fils de cultivateurs et également d'un in. térêt particulier pour cette couche de la société.

Pendant la seconde période (1906-1930), même si les tendances s'atténuent, deux faits retiennent notre attention. Tout d'abord, la proportion d'abandon des fils de cultivateurs fait un bond spectaculaire, passant de $27.6 \%$ à $53 \%$. Ensuite, la proportion totale des abandons, elle aussi, connaît une hausse remarquable, passant de $42 \%$ à $54 \%$. Il apparaît ainsi que la sélection faite par le Séminaire ait été plus rigoureuse, et cela, envers tous les groupes sociaux représentés.

D. Les choix de carrières des étudiants du Séminaire (Cf. Tableaux Va et $\mathrm{Vb})$

Jusqu'à présent, nous nous sommes surtout attachés à étudier le recrutement géographique et social des étudiants et à tenter de déceler, a vec autant de précision que nous le permettaient nos moyens, les facteurs ayant influencé la fréquentation et la persévérance scolaire.

Nous toucherons ici un des principaux objectifs que nous nous étions fixés. Nous allons, en effet, pouvoir rendre compte, par ce qui ressort du Tableau $\mathrm{V}$, de la répartition des choix de carrières des étudiants en regard de leur provenance sociale. Cette partie de notre étude, force nous est de l'admettre, fut certainement la plus difficile à mener à terme, en raison des grandes difficultés rencontrées quand il s'est agi de retracer les orientations professionnels des étudiants.

6 Il faut noter ici l'effort fait par le Séminaire pour tempérer ses exigences financières. On trouva des bienfaiteurs aux étudiants les moins fortunés; on créa l'OEuvre du PetitSéminariste qui servait à dépanner les cas les plus urgents; enfin, l'institution elle-même offrit un genre de prêts à ses étudiants les plus avancés. 
On peut dire toutefois que le nombre de cas que nous sommes parvenus à réunir et l'établissement des proportions auxquelles nous a vons abouti semblent nous offrir une image juste de la réalité.

Le premier cas qui attire l'attention est sans équivoque celui des vocations religieuses. Un regard attentif sur les graphiques nous révèle que cette orientation est de loin la plus choisie puisqu'elle attire, pour chacune des deux périodes, respectivement $70 \%$ et $60 \%$ des finissants. Ce phénomène nous amène à la constatation que le Séminaire semble avoir atteint sans trop de difficultés le principal but qu'il s'était fixé lors de sa fondation.

En deuxième lieu, si on compare l'origine sociale et l'orientation, il est intéressant d'observer la réponse empressée de la classe agricole à l'appel du clergé désireux de perpétuer, à l'intérieur de la société saguenéenne, la religion catholique, grâce à de nombreuses vocations religieuses.

La très forte proportion de fils de cultivateurs optant pour cette orientation éclaire assez bien l'hypothèse que nous avons énoncée précédemment, concernant la sur-représentation des fils de cultivateurs dans la clientèle du Séminaire.

Pour la seconde période (1906-1930), les mêmes remarques peuvent se faire: même si les traits s'atténuent, les cultivateurs sont encore surreprésentés au sein des orientations religieuses et les classes moyennes, sous-représentées. Ce qui transparaît le plus dans l'étude de cette période, c'est l'apparition de nouveaux choix de carrières (Agronomie, Médecine vétérinaire, Enseignement, Industrie et Finance, etc.). Cette diversification correspond peut-être à des préoccupations nouvelles du Séminaire.

Comme conclusion à l'analyse de ce tableau, disons que, massivement, les fils de cultivateurs devenaient prêtres. Voilà fort probablement la raison pour laquelle, même si on diversifiait le recrutement, on les acceptait d'emblée au Séminaire.

\section{CONCLUSION}

En guise de conclusion, disons que, pour la période allant de 1873 1905, notre hypothèse paraît s'avérer exacte et le Séminaire de Chicoutimi ne semble pas s'être lancé dans la voie tracée par les autres institutions d'enseignement classique établies dans notre province. En effet, mettant de côté l'unique objectif de la création d'une élite sociale, il a plutôt tenté d'assurer l'encadrement religieux de la jeune société saguenéenne, en se faisant le grand propagateur de la foi catholique. 
Il nous a semblé que ce choix fondamental, clairement indiqué dans l'échange de correspondance et les déclarations qui ont accompagné la fondation de l'institution, contribue à expliquer la plupart des phénomènes analysés ici. C'est dans le but d'implanter fortement la foi catholique que Monseigneur Racine a voulu fonder un Séminaire destiné à la formation des prêtres : d'où cette prédilection pour la population des campagnes où la moralité et la tradition ont été préservées du modernisme; d'où aussi la sollicitude des pédagogues du Séminaire à l'endroit de la clientèle rurale. On le voit bien par l'étude du recrutement, de la persévérance scolaire et de l'orientation. C'est sans doute parce que les fils de cultivateurs se dirigent en priorité vers la prêtrise qu'on favorise leur recrutement.

La seconde période étudiée ne semble être que le prolongement de la précédente. Toutefois, une observation plus méticuleuse des données nous amène à constater une certaine atténuation des tendances générales de la première période, atténuation qui peut, selon nous, refléter l'amorce d'une mutation des objectifs, servant de préambule à une période de réorientation. En effet, ses priorités culturelles étant assurées, l'institution a très bien pu s'ajoindre d'autres objectifs, s'adjugeant cette fois-ci une fonction proprement sociale. Si tel fut le cas, le Séminaire aurait tenté par la suite de pourvoir les cadres socio-économiques de la région en formant une élite laïque.

La démonstration de cette nouvelle hypothèse nécessitera une étude approfondie de la période faisant suite à 1930 . Néanmoins, dans la mesure où le présent exposé suscitera la curiosité d'autres chercheurs, nos efforts n'auront pas été vains.

Jean-Paul Simard, ptre

D.E.S. en Histoire, professeur d'Histoire

à l'Université du Québec, à Chicoutimi.

Bérard Riverin, D.E.S., Bac. Sp. en Histoire, professeur d'Histoire au CEGEP de Chicoutimi. 
LAC ST. JEAN

ORIGINE RËGIONALE DES ETTUDIANTS

DU SEEMINAIRE DE CHICOUTIMI.

JONQ:KENOOO

chrcouying

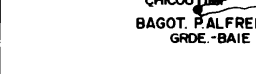
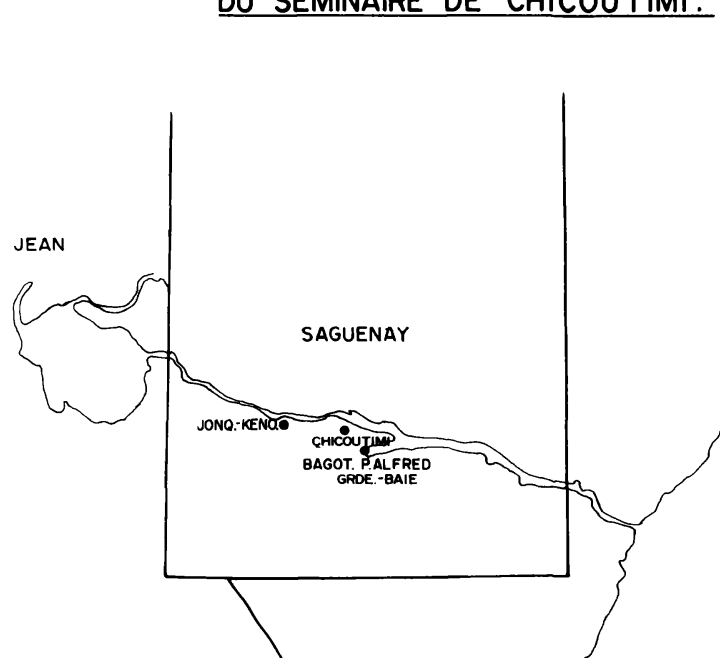

CHARLEVOIX

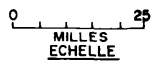


GRILLE CLASSIFICATION SOCIO-PROFESSIONNELLE

\begin{tabular}{|c|c|c|c|c|c|c|c|c|c|c|}
\hline \multirow{2}{*}{$\begin{array}{l}\text { GROUPES } \\
\text { SECTORIELS } \\
\text { CATEGORIES } \\
\text { SOCIO-PROF. }\end{array}$} & \multirow{2}{*}{$\begin{array}{l}\text { CULTIVATEURS } \\
\text { EXPLOITANTS } \\
\text { AGRICOLES }\end{array}$} & \multicolumn{3}{|c|}{ OUVRIERS } & \multicolumn{4}{|c|}{ CLASSES MOYENES } & \multicolumn{2}{|c|}{ ELITE ECONOMIQUE ET SOCIALE } \\
\hline & & $\begin{array}{l}\text { OUVRIRIS } \\
\text { NON-SPECIALISES }\end{array}$ & $\begin{array}{l}\text { OUVRIERS } \\
\text { SPECIALISES }\end{array}$ & $\begin{array}{l}\text { TRAVAILLEURS } \\
\text { FORESTIERS }\end{array}$ & $\begin{array}{l}\text { ARTISANS ET } \\
\text { PETITS ENTREPRE- } \\
\text { NEURS, FABRICANT }\end{array}$ & $\begin{array}{l}\text { METIERS DU } \\
\text { COMMECE } \\
\text { COMERCANTS }\end{array}$ & $\begin{array}{l}\text { METIERS } \\
\text { TERTIAIRES }\end{array}$ & $\begin{array}{l}\text { PETTTS } \\
\text { NOTABLES }\end{array}$ & $\begin{array}{l}\text { PROFESSIONS } \\
\text { LIBERALES }\end{array}$ & $\begin{array}{l}\text { INDUSTRIELS } \\
\text { FINANCLERS } \\
\text { ENTREPRENEURS }\end{array}$ \\
\hline $\begin{array}{l}\text { DEFINITIONS } \\
\text { ATTRIBUEES }\end{array}$ & $\begin{array}{l}\text { Travailleurs de } \\
\text { la terre au sens } \\
\text { très large, } \\
\text { excluant les } \\
\text { jerdiniers et les } \\
\text { aides-fermiers, } \\
\text { se désignant } \\
\text { pour la plupart } \\
\text { coome culti- } \\
\text { vateurs. }\end{array}$ & $\begin{array}{l}\text { Hommes à tout } \\
\text { faire, travail- } \\
\text { leurs saisonniers } \\
\text { employés à des } \\
\text { têches manuelles } \\
\text { n'timpliquant pas } \\
\text { de savoir-faire } \\
\text { très poussé. }\end{array}$ & 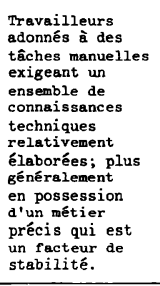 & $\begin{array}{l}\text { Gens vivant } \\
\text { d'une occupation } \\
\text { quelconque en } \\
\text { regard } \bar{a} 1 \mathrm{a} \\
\text { forêt. }\end{array}$ & \begin{tabular}{|l} 
Travailleurs \\
exerçant un \\
métier a leur \\
propre compte, \\
possédant souvent \\
une Echoppe, \\
opérant comme \\
petit contrac- \\
teur, pouvant \\
evoir quelques \\
employés à leur \\
service.
\end{tabular} & $\begin{array}{l}\text { Per sonnes vivant } \\
\text { du petitit négocee } \\
\text { et y exerçant } \\
\text { une occupation. }\end{array}$ & $\begin{array}{l}\text { Employés de } \\
\text { services, petits } \\
\text { cadres de 1'ad- } \\
\text { ministration } \\
\text { locale. }\end{array}$ & $\begin{array}{l}\text { Occupations } \\
\text { impliquant } \\
\text { l'exercice } \\
\text { d'une autorité } \\
\text { ou d'un lea- } \\
\text { dership; pos- } \\
\text { tes à caractère } \\
\text { prestigieux ou } \\
\text { honorifique. }\end{array}$ & $\begin{array}{l}\text { Professions } \\
\text { intellectuelles } \\
\text { au sens très } \\
\text { large: Couche } \\
\text { supérieure de } \\
\text { la notabilité, } \\
\text { de l'élite } \\
\text { professionnelle. }\end{array}$ & $\begin{array}{l}\text { Les personns- } \\
\text { ges importants; } \\
\text { les couches } \\
\text { supérieures de } \\
\text { l'érite écono- } \\
\text { mique. }\end{array}$ \\
\hline $\begin{array}{l}\text { LISTEE DES } \\
\text { OCCUPATIONS } \\
\text { ENGLOBERS }\end{array}$ & $\begin{array}{l}\text { Colon (excepti- } \\
\text { onnellement) } \\
\text { Cultivateur } \\
\text { Fermier }\end{array}$ & $\begin{array}{l}\text { Aide de ferme } \\
\text { Comnis } \\
\text { Concierge } \\
\text { Cuviste } \\
\text { Domestique } \\
\text { Engage } \\
\text { Gardien } \\
\text { Journalier } \\
\text { Livreur } \\
\text { Manouvre } \\
\text { Ménagère } \\
\text { ouvrier } \\
\text { Pompiste } \\
\text { Serveuse } \\
\text { Etc. }\end{array}$ & $\begin{array}{l}\text { Aiguilleur } \\
\text { Analyste } \\
\text { Barman } \\
\text { Briqueteur } \\
\text { Camionneur } \\
\text { Charpentier } \\
\text { Charron } \\
\text { Chaurfeur } \\
\text { Conducteur } \\
\text { Couturier } \\
\text { Cuisinier } \\
\text { Debosseur } \\
\text { Dessinateur } \\
\text { Electricien } \\
\text { Imprimeur } \\
\text { Infirmier } \\
\text { Jaridinier } \\
\text { Machiniste } \\
\text { Maçon } \\
\text { Mecanicien } \\
\text { Menuisier } \\
\text { Monteur } \\
\text { Opérateur } \\
\text { Peintrere } \\
\text { Plombier } \\
\text { Soudeur } \\
\text { Technicien } \\
\text { Etc. }\end{array}$ & $\begin{array}{l}\text { Bûcheron } \\
\text { Garde-feu } \\
\text { Garde-chasse et } \\
\text { pêche } \\
\text { Garde-forestier } \\
\text { Homme de } \\
\text { chantier } \\
\text { Etc. }\end{array}$ & \begin{tabular}{|l} 
Arpenteur \\
Barbier \\
Beurrier \\
Boucher \\
Boulanger \\
Charretier \\
Cocher \\
Coiffeur \\
Confiseur \\
Contracteur \\
Cordonnier \\
Crémier \\
Dérorateur \\
merbouteilleur \\
Entrepreneur de \\
pompes funebres \\
Forgeron \\
Foromager \\
Garagiste \\
Imprimeur \\
Laitier \\
Manufacturier \\
Maunier \\
Modiste \\
Navigateur \\
Pêcheur \\
Perruquier \\
Tailleur \\
Tanneur \\
Etc.
\end{tabular} & $\begin{array}{l}\text { Agent } \\
\text { Bijoutier } \\
\text { Commerçant } \\
\text { Commis-voya- } \\
\text { geur s -voug } \\
\text { Comptable } \\
\text { Courtier } \\
\text { Epicier } \\
\text { Gérant } \\
\text { Hôntelier } \\
\text { Marchand } \\
\text { Négociant } \\
\text { Représentent } \\
\text { Restaurateur } \\
\text { Vendeur } \\
\text { Etc. }\end{array}$ & $\begin{array}{l}\text { Assureur } \\
\text { Bibliothécaire } \\
\text { Caissier } \\
\text { Contrôleur } \\
\text { Rmployé civil } \\
\text { Fonctionaire } \\
\text { Huissier } \\
\text { Percepteur } \\
\text { Secrétaire } \\
\text { Télégraphiste } \\
\text { Teneur de } \\
\text { livres } \\
\text { Trésorier } \\
\text { Vérificateur } \\
\text { Etc. }\end{array}$ & $\begin{array}{l}\text { Chef de gare } \\
\text { Conferencier } \\
\text { Greffier } \\
\text { Inspecteur } \\
\text { d'école } \\
\text { Instituteur } \\
\text { Journaliste } \\
\text { Maitre de } \\
\text { poste } \\
\text { Policier } \\
\text { Professeur } \\
\text { Sacristain } \\
\text { Sherrif } \\
\text { Etc. }\end{array}$ & $\begin{array}{l}\text { Agronome } \\
\text { Avocat } \\
\text { Coroner } \\
\text { Ingénieur } \\
\text { Juge } \\
\text { Magistrat } \\
\text { MEgdecin } \\
\text { Notaire } \\
\text { Procureur } \\
\text { Protonotaire } \\
\text { Etc. }\end{array}$ & $\begin{array}{l}\text { Banquier } \\
\text { Contracteur (gros) } \\
\text { Courtier } \\
\text { Entrepreneur(gros) } \\
\text { Gérant de } \\
\text { Banque } \\
\text { Industriel } \\
\text { Etc. }\end{array}$ \\
\hline
\end{tabular}


Tableau $\mathrm{n}^{\circ} \mathrm{I}$

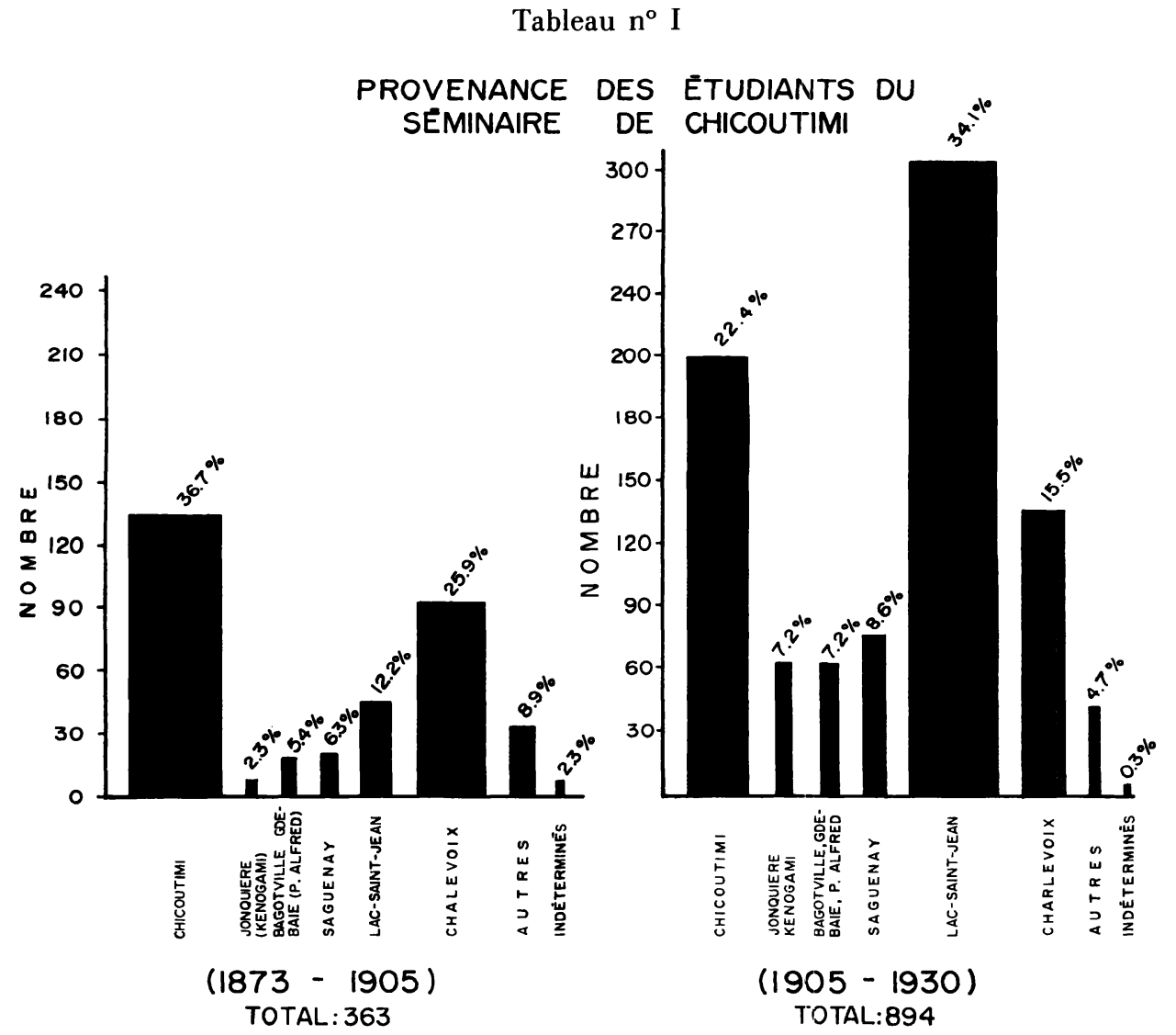


Tableau $n^{\circ}$ II

FREQUENTATION SCOLAIRE ET ORIGINE SOCIALE
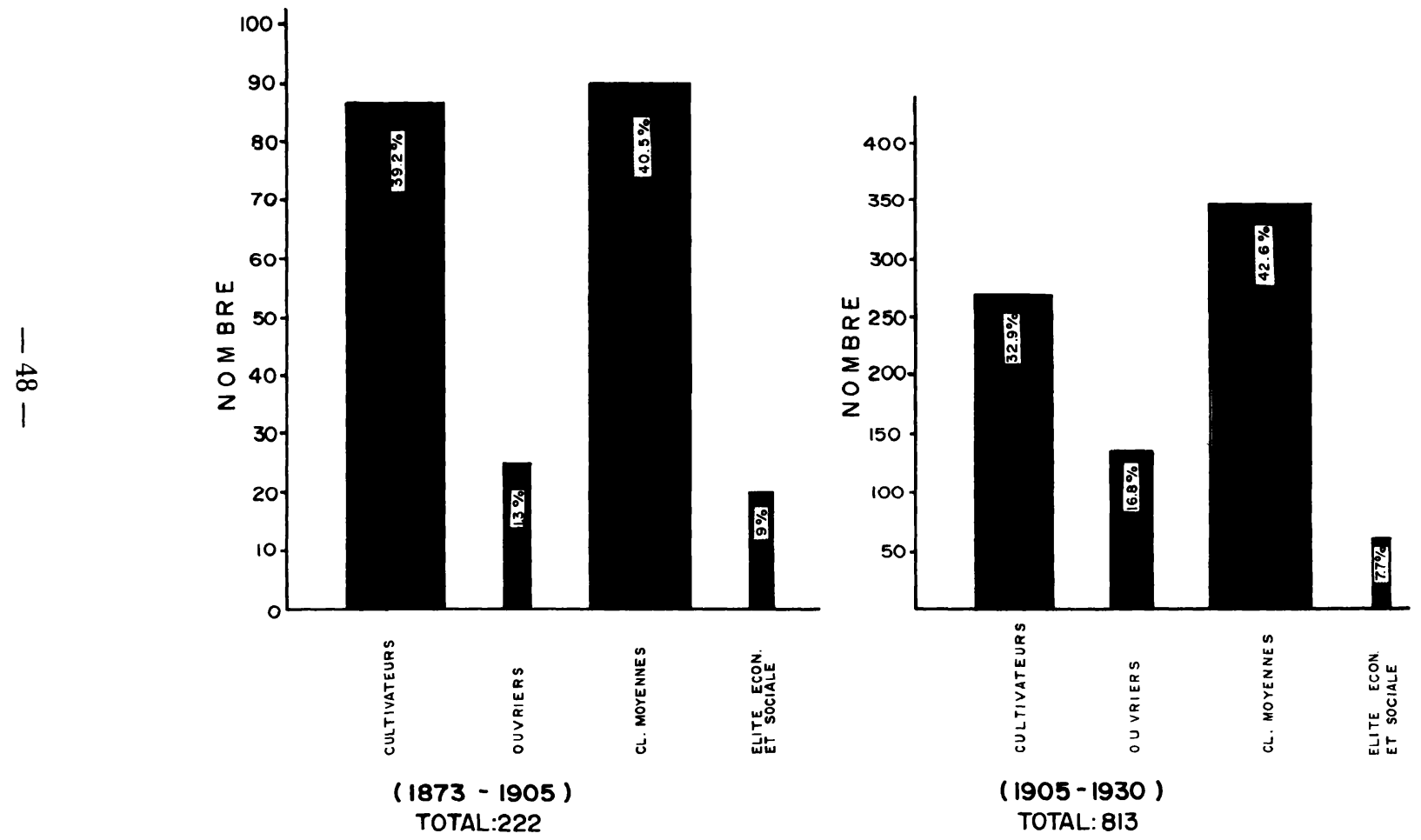
Tableau $\mathrm{n}^{\circ}$ III

FACTEURS SOCIAUX DE LA
PERSEEVERANCE SCOLAIRE

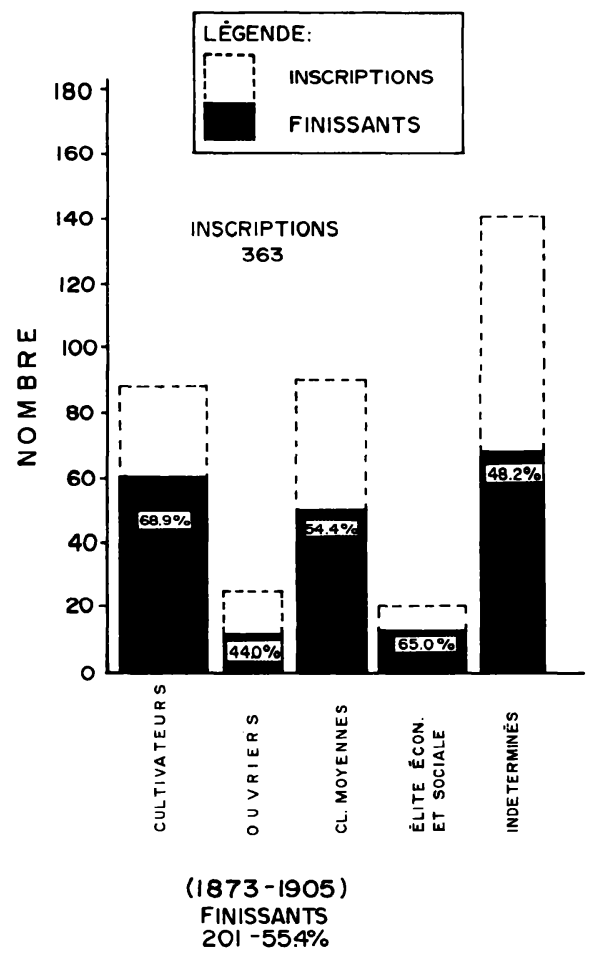

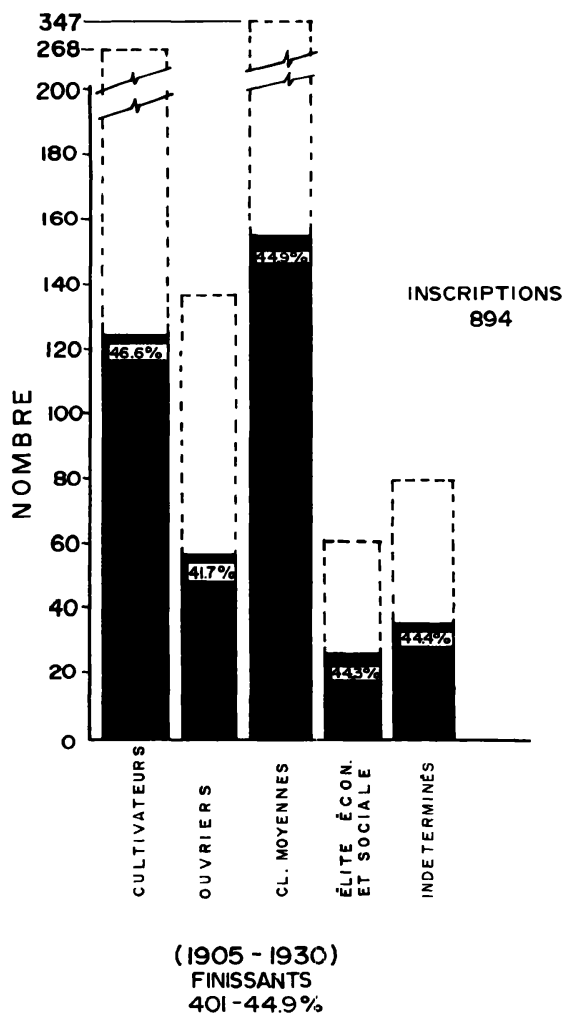


Tableau $n^{0}$ IVa

\section{LES ABANDONS (1873-1905) TOTAL: $153-42 \%$}
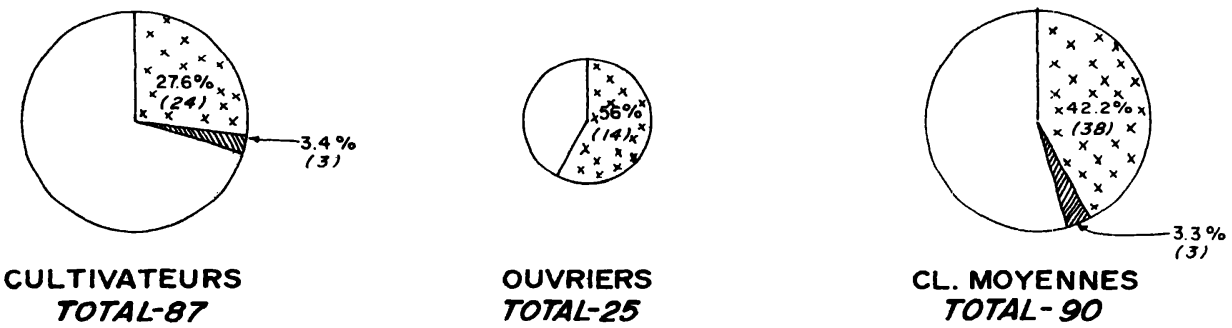

en

$$
\text { TOTAL-8T }
$$

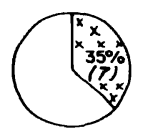
ËLITE ÉCON. ET SOCIALE TOTAL-2O

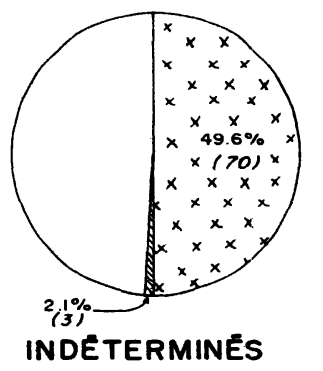

TOTAL-14I
LËGENDE:

ABANDONS

DECES 
Tableau $n^{\circ}$ IVb

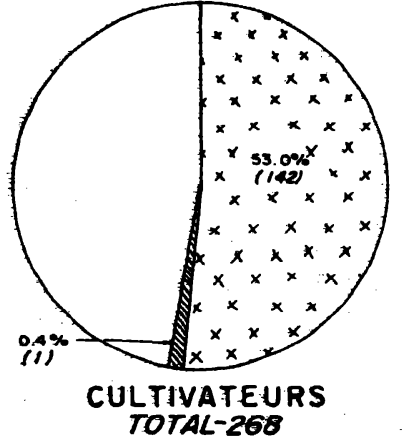

\section{LES ABANDONS}

(1905-1930)

TOTAL: $483-54 \%$

LEGENDE:

ABANDONS

DITOONO DECES

TOTAL-268
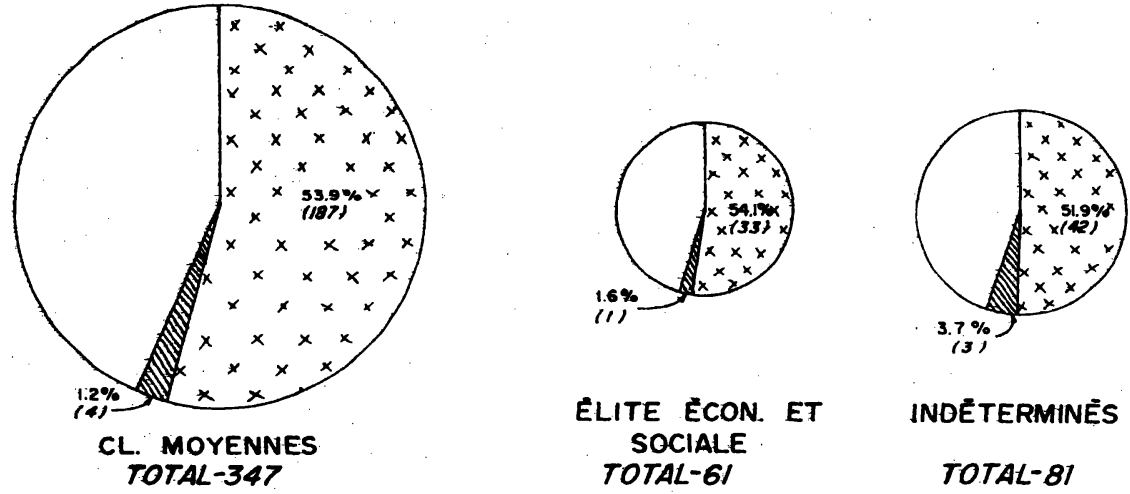

ELITE ECON. ET SOCIALE TOTAL-GI

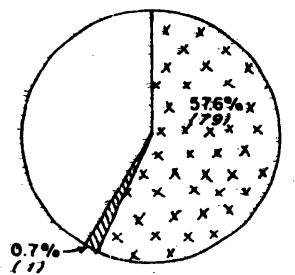

OUVRIERS

TOTAL-13T

$$
\text { TOTAL-347. }
$$

TOTAL- 81 
Tableau $n^{\circ}$ Va

CHOIX DES CARRIÉRES ET ORIGINE SOCIALE

$(1873-1905)$

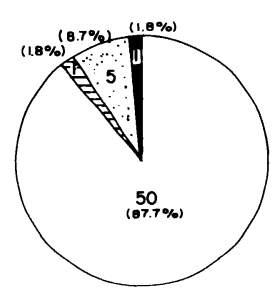

CULTIVATEURS

57

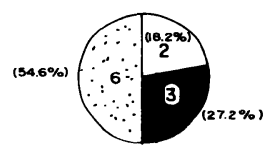

ÉLITE ECONOMIQUE ET SOCIALE II

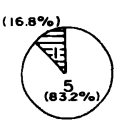

OUVRIERS

6

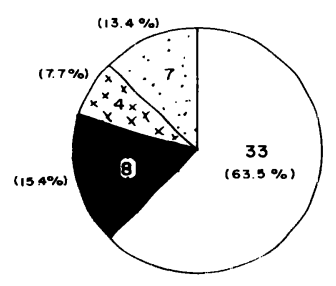

INDE TERMINES

52

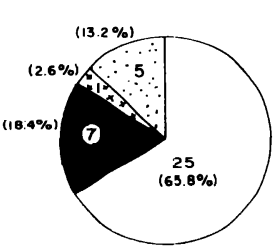

CL. MOYENNES

38

RELIGIEUX

SC. SANTE

PROF. JURIDIQUES

COMMERCE

GÉNIE -ARPENTAGE 
Tableau $\mathrm{n}^{\circ} \mathrm{Vb}$

\section{CHOIX DES CARRIËRES ET ORIGINE SOCIALE}

$(1905-1930)$
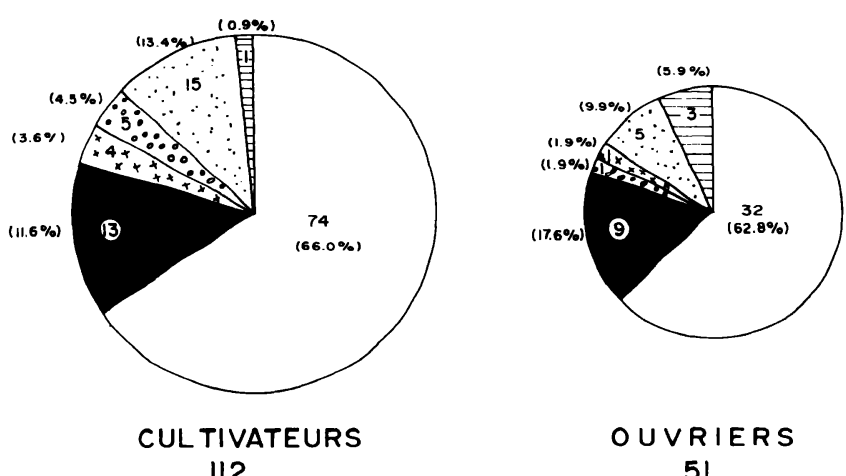

OUVRIERS

51

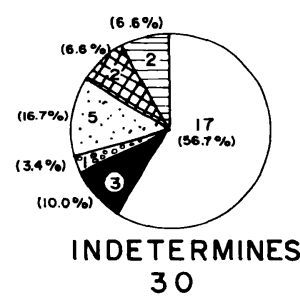

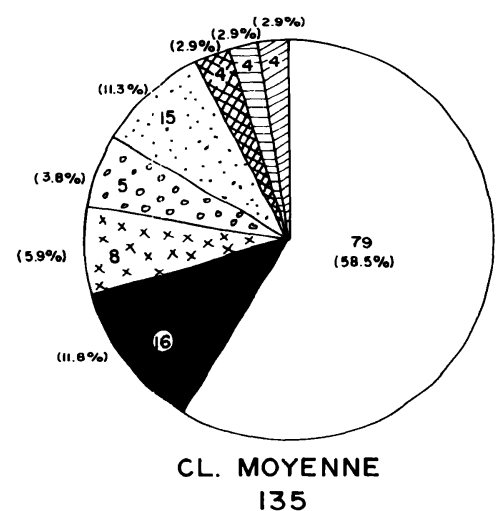

$\square$ Religieux

$\longrightarrow$ SC. SANTE

COMMERCE

$\because$ AGRON. VEETEER.

PROF. JURIDIQUE

ENSEIGNEMENT

$\Longrightarrow$ GÉNIE-ARPENTAGE

INDUST. FINANCE ENT. 